\title{
Diffusing-wave spectroscopy in a shear flow
}

\author{
X-L. Wu \\ Exxon Research and Engineering, Annandale, New Jersey 08801 \\ D. J. Pine \\ Department of Physics, Haverford College, Haverford, Pennsylvania 19041 \\ P. M. Chaikin, J. S. Huang, and D. A. Weitz \\ Exxon Research and Engineering, Annandale, New Jersey 08801
}

Received June 16, 1989; accepted September 28, 1989

\begin{abstract}
We present a new technique for measuring velocity gradients for laminar shear flow, using dynamic light scattering in the strongly multiple-scattering regime. We derive temporal autocorrelation functions for multiply scattered light, taking into account particle displacements arising from deterministic shear flow and random Brownian motion. The laminar shear flow and Brownian motion are characterized by the relaxation rates $\tau_{S}^{-1}=\bar{\Gamma} k_{0} l^{*} / \sqrt{30}$ and $\tau_{B}{ }^{-1}=D k_{0}{ }^{2}$, respectively, where $\bar{\Gamma}$ is the mean shear rate of the scatterers, $k_{0}=2 \pi n / \lambda$ is the wave number in the scattering medium, $l^{*}$ is the transport mean free path of the photons, and $D$ is the diffusion coefficient of the scatterers. We obtain excellent agreement between theory and experiment over a wide range of shear rates, 0.5 $\sec ^{-1}<\bar{\Gamma}<200 \mathrm{sec}^{-1}$. In addition, the autocorrelation function for forward scattering is independent of the scattering properties of the medium and depends only on the mean shear rate and sample thickness when $\tau_{S}$ is much less than $\tau_{B}$. Thus the mean shear rate can be simply determined by a single measurement.
\end{abstract}

\section{INTRODUCTION}

The measurement of the flow of fluids is critical to a wide range of studies of both technological importance and fundamental interest. While a variety of experimental techniques have been employed, various forms of dynamic light scattering (DLS) have become increasingly widely used. Light scattering is both accurate and relatively simple and provides a nonintrusive measure of the fluid flow. The only requirement for the application of light-scattering techniques is that the fluid contain a low concentration of small particles that serve as markers by flowing with the fluid and scattering the laser light. Uniform flow must be measured by a beating or heterodyne technique, which entails the use of a second laser beam to provide a constant reference frequency. By contrast, homodyne techniques, which employ only a single beam, are sensitive only to the relative velocities of the scattering particles. Thus these techniques are useful for measuring velocity gradients or shear $^{1}$ or for studying turbulence. ${ }^{2}$

A major limitation of these dynamic light-scattering techniques is the requirement that the scattering particles be maintained at a low concentration. This is necessary both to allow the laser beam to propagate through the liquid and to ensure that only single scattering occurs. This severely limits the application of these techniques and precludes their use for studying many potentially interesting and important systems, such as dense colloidal suspensions ${ }^{3,4}$ and blood flow in tissues. ${ }^{5}$ In this paper we introduce a new technique that overcomes some of these limitations. It is ideally suited to the study of the flow of turbid fluids in which the light is strongly scattered. We limit ourselves to homodyne scattering and therefore consider only the measurement of relative velocities.

The key to the new technique is the description of the propagation of light in a strongly scattering medium in terms of a random walk. Thus the transport of light is assumed to be diffusive. ${ }^{6}$ The photon-diffusion approximation has recently been exploited to develop an expression for the temporal fluctuations of the intensity of the scattered light and has been applied to study the Brownian motion in optically thick suspensions. ${ }^{7}$ This technique is called diffusing wave spectroscopy ${ }^{8}$ (DWS) and allows the more traditional techniques of DLS to be extended to strongly multiple-scattering media.

In this paper we extend the DWS theory to include the case when the scatterers are subjected to a laminar shear flow in addition to their own Brownian motion. We find that there are two competing dynamical processes in this problem, each having its own characteristic time dependence. For a stochastic process the square of Brownian particle displacement $\left\langle\Delta r^{2}(\tau)\right\rangle$ is proportional to time, $\tau$, and for a deterministic motion $\left\langle\Delta r^{2}(\tau)\right\rangle$ is proportional to $\tau^{2}$. Associated with these processes are two characteristic times, the Brownian diffusion time, $\tau_{B}$, and the shear relaxation time, $\tau_{S}$. The autocorrelation functions that we derive correctly reflect the interplay between these two time scales. Using an optically thick suspension of uniformly sized polystyrene spheres, we find that the measured autocorrelation functions for both forward and backward scattering are in good agreement with our theory over a wide range of shear rates. Thus it is possible to extend DLS to the strongmultiple-scattering regime where the system dynamics has more than one time scale. 


\section{THEORY}

As in all DLS experiments, the motion of particles is probed by monitoring the time dependence of the fluctuations of the scattered light. Thus we must determine the temporal autocorrelation function $G_{1}(\tau)=\langle E(0) E(\tau)\rangle$, where $E(\tau)$ is the electric field of the scattered light that is collected by the detector. To calculate $G_{1}(\tau)$, we consider the light, which is multiply scattered by a random distribution of particles, to execute a random walk through the sample. In the limit that the scattering mean free path, $l$, is much larger than the wavelength of light but much smaller than the linear dimension of a sample cell, the intensity of light leaving the sample will be the incoherent sum of the electric field of light scattered through all possible paths. ${ }^{9}$ Thus, for a given light path consisting of a sequence of $n$ scattering events at positions $\mathbf{r}_{1}, \ldots, \mathbf{r}_{n}$ and with corresponding successive wavevector transfers $\mathbf{q}_{i}=\mathbf{k}_{i}-\mathbf{k}_{i-1}$, the (scalar) electric field at time $\tau$ will be $E^{(n)}(\tau) \simeq\left|E^{(n)}(0)\right| \exp \left[-i \sum_{i=1}^{n} \mathbf{q}_{i} \cdot \mathbf{r}_{i}(\tau)\right]$. The dynamical information about the scatterers that is probed by $G_{1}(\tau)$ is contained in the phase difference $\Phi^{(n)}(\tau)$ between $E^{(n)}(0)$ and $E^{(n)}(\tau)$ and can be written as $\Phi^{(n)}(\tau)=\sum_{i=1}^{n} \Phi_{i}(\tau)$ $=\sum_{i=1}^{n} \mathbf{q}_{i} \cdot \Delta \mathbf{r}_{i}(\tau)$, where $\Delta \mathbf{r}_{i}(\tau)$ is the change in position of the $i$ th scatterer during the time interval $\tau$. If the Brownian motion of particles is not affected by the laminar shear flow, ${ }^{10}$ then $\Phi^{(n)}(\tau)=\sum_{i=1}^{n} \mathbf{q}_{i} \cdot\left[\Delta \mathbf{r}_{i}^{B}(\tau)+\Delta \mathbf{r}_{i}^{S}(\tau)\right]$, where $\Delta \mathbf{r}_{i}^{B}(\tau)$ and $\Delta \mathbf{r}_{i}^{S}(\tau)$ are the displacements of particle $i$ from particle diffusion and convective shear, respectively. Since we are interested only in relative motions between particles for the shear motion, we can decompose $\mathbf{q}_{i}$ to give $\sum_{i=1}^{n} \mathbf{q}_{i} \cdot$ $\Delta \mathbf{r}_{i}^{S}(\tau)=\sum_{i=1}^{n} \mathbf{k}_{i} \cdot\left[\Delta \mathbf{r}_{i+1} S(\tau)-\Delta \mathbf{r}_{i}^{S}(\tau)\right]$. Then the total phase shift is simply ${ }^{11}$

$$
\Phi^{(n)}(\tau)=\sum_{i=1}^{n}\left\{\mathbf{q}_{i} \cdot \Delta \mathbf{r}_{i}^{B}(\tau)+\mathbf{k}_{i} \cdot\left[\Delta \mathbf{r}_{i+1}^{S}(\tau)-\Delta \mathbf{r}_{i}^{S}(\tau)\right]\right\}
$$

The simplest case to treat is that of planar Couette flow. Therefore we take a velocity profile given by $\mathbf{v}_{x}=\Gamma z \hat{e}_{x}$ as shown in Fig. 1, where $\Gamma=\partial v_{x} / \partial z$ is the rate of shear and $\hat{e}_{x}$ is a unit vector in the $x$ direction. Then the contribution to the time-dependent phase due to shear for the $i$ th scattering event is $\mathbf{k}_{i} \cdot\left[\Delta \mathbf{r}_{i+1} S(\tau)-\Delta \mathbf{r}_{i}{ }^{S}(\tau)\right]=k_{0} \hat{\kappa}_{i} \cdot\left[\Gamma\left(\Lambda_{i} \hat{\kappa}_{i} \cdot \hat{e}_{z}\right) \hat{e}_{x} \tau\right]=$

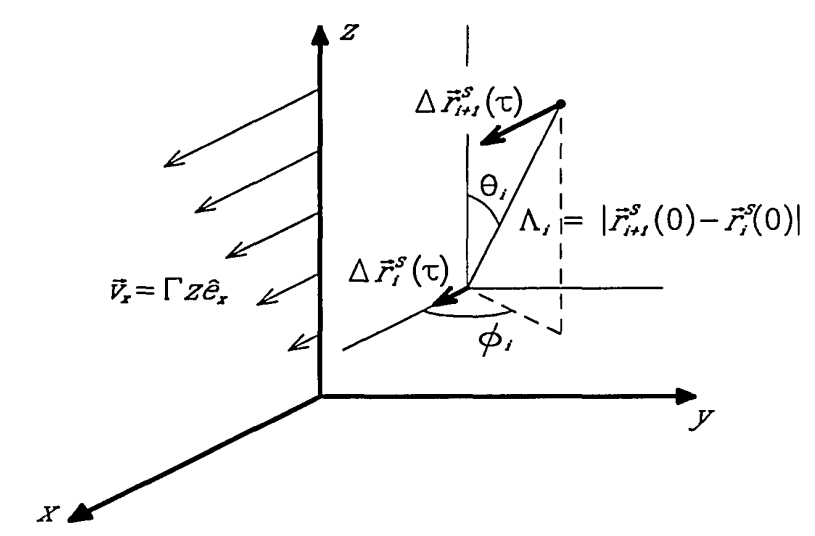

Fig. 1. Geometry for the calculation of the phase shift due to shear for the $i$ th scattering event: The flow is in the $x$ direction, and the velocity gradient, $\Gamma$, is in the $z$ direction. The distance between successive events is $\Lambda_{i}=\left|\mathbf{r}_{i+1}(0)-\mathbf{r}_{i}(0)\right|$, and the change in position of $i$ th scatterer in the time interval $\tau$ owing to convective shear is $\Delta \mathbf{r}_{i}^{S}(\tau)$.
$\Gamma \tau k_{0} \Lambda_{i}\left(\hat{\kappa}_{i} \cdot \hat{e}_{x}\right)\left(\hat{\kappa}_{i} \cdot \hat{e}_{z}\right)$, where $k_{0}=2 \pi \mathrm{n} / \lambda$ is the wave number in the scattering medium, $\Lambda_{i}=\left|\mathbf{r}_{i+1}(0)-\mathbf{r}_{i}(0)\right|$ is the distance between scattering events, $\hat{\kappa}_{i}$ is a unit vector in the direction of the scattered light, and $\hat{e}_{z}$ is a unit vector in the $z$ direction. This formula can be rewritten in terms of polar and azimuthal angles $\theta_{i}$ and $\phi_{i}$ :

$$
\mathbf{k}_{i} \cdot\left[\Delta \mathbf{r}_{i+1} S(\tau)-\Delta \mathbf{r}_{i}^{S}(\tau)\right]=\Gamma \tau k_{0} \Lambda_{i} \cos \theta_{i} \sin \theta_{i} \cos \phi_{i},
$$

where $\theta_{i}$ and $\phi_{i}$ are defined in Fig. 1.

The heterodyne autocorrelation function $G_{1}{ }^{(n)}(\tau)$ for a given multiple-scattering path of order $n$ can then be written as

$$
G_{1}^{(n)}(\tau)=\left\langle E^{(n)}(0) E^{*(n)}(\tau)\right\rangle=\left\langle\left|E^{(n)}(0)\right|^{2}\right\rangle\left\langle\prod_{i=1}^{n} \exp \left[-i \Phi_{i}(\tau)\right]\right\rangle
$$

Therefore the average contribution of all paths of order $n$ is given by

$$
G_{1}^{(n)}(\tau)=I_{0} P(n)\left\langle\prod_{i=1}^{n} \exp \left[-i \Phi_{i}(\tau)\right]\right\rangle
$$

where $P(n)$ is the fraction of total scattering intensity $I_{0}$ in the $n$ th-order paths. Assuming that fields belonging to different paths add incoherently, $G_{1}(\tau)$ is obtained by summing over all possible $n$ :

$$
G_{1}(\tau)=I_{0} \sum_{i=1}^{\infty} P(n)\left\langle\prod_{i=1}^{n} \exp \left[-i \Phi_{i}(\tau)\right]\right\rangle .
$$

For small particles that scatter light isotropically, the successive $\Phi_{i}(\tau)$ are uncorrelated, and the average of the product in Eq. (5) becomes $\left\langle\exp \left[-i \Phi_{i}(\tau)\right]\right\rangle^{n}$, where $\langle\ldots\rangle$ denotes both the configurational average of $\Delta \mathbf{r}_{i}(\tau)$ and the average over all possible scattering vectors $\mathbf{q}_{i}$ and $\mathbf{k}_{i}$ (and hence $\phi_{i}$ and $\theta_{i}$ as defined in Fig. 1). This gives

$$
G_{1}(\tau) \simeq I_{0} \sum_{n=1}^{\infty} P(n)\left\langle\exp \left[i \Phi_{i}(\tau)\right]\right\rangle^{n}
$$

Since $\Delta \mathbf{r}_{i}^{B}(\tau)$ and $\Delta \mathbf{r}_{i}^{S}(\tau)$ represent two independent motions, the configurational average over each of them can be performed separately. For Brownian motion, the derivation can be found in Refs. 7 and 8. For the convective shear part, we make a moment expansion. Since the leading nonvanishing term in such an expansion is the second moment, we have approximately $\langle\ldots\rangle=\exp \left\{-2\left[\tau / \tau_{B}+\left(\tau / \tau_{S}\right)^{2}\right\}\right.$. Here $\tau_{B}{ }^{-1}=D k_{0}^{2}$ and $\tau_{S}{ }^{-1}=\Gamma l k_{0} / \sqrt{30}$, where $D$ is a diffusion constant of the scatterers and $l=\langle\Lambda\rangle$ is the scattering mean free path for light. The factor of $\sqrt{30}$ in $\tau_{S}$ is obtained by averaging $\sin ^{2} 2 \theta \cos ^{2} \phi$ over the unit sphere. For inhomogeneous velocity gradients, $\Gamma^{2}$ must be averaged over the volume of fluid probed by the scattered light that is collected by the detector. Thus $\Gamma$ is replaced by $\bar{\Gamma}=\sqrt{\left\langle\Gamma^{2}\right\rangle}$ in the expression for $\tau_{S}$. The mean free path, $l$, sets the length scale over which $\bar{\Gamma}$ is measured. In the continuum limit we approximate the sum over $n$ in relation (6) by an integral over the scattered-light path length $s=n l$ :

$$
G_{1}(\tau)=I_{0} \int P(s) \exp \left\{-2\left[\tau / \tau_{B}+\left(\tau / \tau_{S}\right)^{2}\right] s / l\right\} \mathrm{d} s,
$$


where $P(s)$ is now the fraction of the total scattered intensity that traverses a path of length $s$ through the sample to the point where the light is detected.

We note that the numerical factor of $\sqrt{30}$ applies to pure elongational flow, as well as to simple Couette flow, so long as the flow is two dimensional. This is because linear shear flow can always be decomposed into an elongational flow and a rotational flow. ${ }^{12}$ Since rotational flow is equivalent to a solid rotation, the relative positions between particles are fixed, and there is no contribution to the decay of the autocorrelation function, except for the initial and final scattering events at the entrance and exit points of the light. Since the total number of scattering events is assumed to be large, the contributions of these two scattering events are small and can be neglected. Thus, in the multiple-scattering regime, elongational and simple shear flow have the same effect on the autocorrelation function.

For larger particles, which scatter light anisotropically, the results are exactly the same, provided that $l$ is everywhere replaced by $l^{*}$, the transport mean free path. Here $l$ and $l^{*}$ are related through the particle form factor $F(\mathbf{q})$ (Ref. 13):

$$
l^{*} / l=\int F(\mathbf{q}) \mathrm{d} \mathbf{q} / \int F(\mathbf{q})(1-\cos \theta) \mathrm{d} \mathbf{q} .
$$

Thus, in general,

$$
G_{1}(\tau)=I_{0} \int P(s) \exp \left\{-2\left[\tau / \tau_{B}+\left(\tau / \tau_{S}\right)^{2}\right] s / l^{*}\right\} \mathrm{d} s,
$$

where $\tau_{S}{ }^{-1}=\Gamma k_{0} l^{*} / \sqrt{30}$. Reference 8 reports that a similar result was obtained for the case of diffusing without shear $\left(\tau_{S} \rightarrow \infty\right)$, and $G_{1}(\tau)$ was given for several geometries of interest in scattering experiments.

It is important to note that for laminar shear flow $G_{1}(\tau)$ consists of two independent time scales, $\tau_{B}$ and $\tau_{S}$, corresponding to Brownian motion and convective shear flow, respectively. In the absence of shear, $G_{1}(\tau)$ has an exponential decay of $\exp \left(-2 \tau / \tau_{B}\right)$ per scattering event, which is characteristic of stochastic motion. For a system in which Brownian diffusion can be neglected, $G_{1}(\tau)$ has a Gaussian decay of $\exp \left[-2\left(\tau / \tau_{S}\right)^{2}\right]$ per scattering event, which is characteristic of convective motion. In general, the decay of $G_{1}(\tau)$ is dominated by Brownian motion for $\tau<\left(\tau_{S} / \tau_{B}\right) \tau_{S}$ and by shear flow for $\tau>\left(\tau_{S} / \tau_{B}\right) \tau_{S}$.

The key to the solution of Eq. (7) is the determination of $P(s)$ for a given experimental geometry. To this end, we assume that the transport of light through the sample is diffusive so that the density of diffusing photons, $U$, is described by the diffusion equation, $\partial U / \partial t=D_{l} \nabla^{2} U$, where $D_{l}$ $=c l^{*} / 3$ is the diffusion coefficient of light. We take as the source of diffusing intensity a point a distance $\gamma l^{*}$ inside the sample, where $\gamma \simeq 2$, and set $U=0$ at the boundaries. ${ }^{8}$ As described in Ref. $8, P(s)$, and hence $G_{1}(\tau)$, can be determined from the solution to the photon-diffusion equation for the experimental geometry of interest. However, our task is made simpler by noting that the form of Eq. (7) is identical to the results obtained in Ref. 8 for the case of diffusion without shear, provided that we make the simple substitution $\tau / \tau_{B} \rightarrow\left[\tau / \tau_{B}+\left(\tau / \tau_{S}\right)^{2}\right]$. Thus we can simply adapt the results of Ref. 8 to the case of laminar shear flow.

For forward scattering with a point source on axis with the detector we obtain

$$
G_{1}(\tau)=\frac{L}{\gamma l^{*} \zeta(3)} \int_{\frac{L}{l^{*}}\left\{6\left[\tau / \tau_{B}+\left(\tau / \tau_{S}\right)^{2}\right]\right\}^{1 / 2}}^{\infty} \frac{z \sinh \left(\gamma l^{*} z / L\right)}{\sinh (z)} \mathrm{d} z
$$

where $\zeta(3) \simeq 4.202$ is the third Riemann zeta function and $L$ is the thickness of the sample cell (here $L=a$ ). Thus for forward scattering the characteristic time for $G_{1}(\tau)$ to decay is $\left(l^{*} / L\right)^{2} \tau_{B}$ if $\tau_{B} \ll \tau_{S}$ and $\left(l^{*} / L\right) \tau_{S}$ if $\tau_{B} \gg \tau_{S}$.

For backscattering with an extended plane-wave source we obtain

$$
G_{1}(\tau)=\frac{1}{1-\gamma l^{*} / L} \frac{\sinh \left(\frac{L}{l^{*}}\left\{6\left[\tau / \tau_{B}+\left(\tau / \tau_{S}\right)^{2}\right]\right\}^{1 / 2}\left(1-\gamma l^{*} / L\right)\right)}{\sinh \left(\frac{L}{l^{*}}\left\{6\left[\tau / \tau_{B}+\left(\tau / \tau_{S}\right)^{2}\right]\right\}^{1 / 2}\right)} .
$$

In the limit of $L / l^{*} \gg 1, G_{1}(\tau)$ decays exponentially as $\sqrt{\tau / \tau_{B}}$ if $\tau_{B} \ll \tau_{S}$ or as $\tau / \tau_{S}$ if $\tau_{B} \gg \tau_{S}$.

These autocorrelation functions obtained for the case of multiple scattering decay much more quickly than autocorrelation functions obtained in the single-scattering limit. Thus the distance that a typical scatterer moves in a decay time is also shorter. We can estimate this distance by noting that the autocorrelation function decays significantly when particle motion causes the photon path length to change by $\sim \lambda$. In this case the mean-square total phase shift due to particle motion is unity, $\left[\Phi^{(n)}\right]^{2} \simeq n k_{0}{ }^{2}\left\langle(\Delta \mathbf{r})^{2}\right\rangle \simeq 1$, where $n$ is the number of scattering events. Thus we estimate the typical rms displacement $\Delta r_{\mathrm{rms}} \simeq \lambda /(2 \pi \sqrt{n})$. Since $n \gg 1$, $\Delta r_{\mathrm{rms}}$ is much smaller than the wavelength of light. In the single-scattering limit, $\Delta r_{\text {rms }} \simeq \lambda$.

In our experiments, we measure the intensity-intensity autocorrelation function $\langle I(\tau) I(0)\rangle /\langle I(0)\rangle^{2}=1+f(A) g_{2}(\tau)$, where $f(A)$ is a constant determined largely by the collection optics and $g_{2}(\tau)$, the homodyne signal, is related to $G_{1}(\tau)$ by the Siegert relation ${ }^{14}$

$$
g_{2}(\tau)=G_{2}(\tau) / G_{2}(0)=\left|G_{1}(\tau) / G_{1}(0)\right|^{2}
$$

\section{EXPERIMENTS}

To test these ideas, we performed experiments in a rectangular flow cell with the sample dimensions shown in Fig. 2. Because of the large aspect ratio of the sample cell, $b / a=12$, the dominant velocity gradient was in the $z$ direction. We confirmed this by performing a DLS experiment in the single-scattering regime, where we found that measured autocorrelation functions did not change as the sample cell was translated along the $y$ axis except when the laser beam was within approximately $0.1 \mathrm{~cm}$ of the top or the bottom of the sample cell. For a given flow rate, $J$, it can be shown ${ }^{15}$ that the velocity profile in the $z$ direction is a parabolic, so that the shear rate is a linear function of $z, \Gamma(z)=8 V_{\max } z / a^{2}(-a /$ $2<z<a / 2$ ), where $V_{\max }=3 J / 2 a b$ is the velocity in the center of the sample cell. In order to make a comparison with the theoretical calculations we have used a spatially averaged rms shear rate $\bar{\Gamma}=\sqrt{\left\langle\Gamma^{2}\right\rangle}=3 J / a^{2} b$ for our experiment.

A suspension of uniform-diameter polystyrene spheres ${ }^{16}$ (PSS's) $(0.415 \pm 0.005 \mu \mathrm{m})$ at a volume fraction of $2 \%$ was 


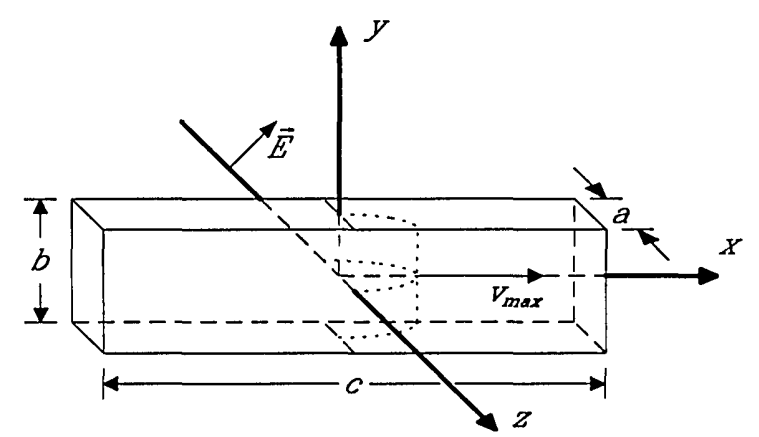

Fig. 2. Sample geometry: The cell was made of quartz, which has the following dimensions: $a=0.1 \mathrm{~cm}, b=1.2 \mathrm{~cm}$, and $c=50 \mathrm{~cm}$. A suspension of $0.415-\mu \mathrm{m}$-diam PSS's at a volume fraction of $2 \%$ was pumped through the cell with a $50-\mathrm{cm}^{3}$ syringe. The resulting velocity profiles with $V_{\max }$ at the front are shown. The incident laser beam, directed along the $z$ axis and polarized at $45 \mathrm{deg}$ with respect to the $x$ axis, passed through the center of the cell, where the only velocity gradient was in $z$ direction.

pumped through the cell at a constant rate, using a $50-\mathrm{cm}^{3}$ syringe and a Sage pump (Model 355). A maximum pumping rate of $J_{\max }=0.75 \mathrm{~cm}^{3} / \mathrm{sec}$ could easily be achieved with this apparatus. At this pumping rate a maximum velocity of $11 \mathrm{~cm} / \mathrm{sec}$ was obtained in the middle of the cell, which corresponds to a Reynolds number $R e=V_{\max } a / \nu \simeq 100$. For Poiseuille flow this Reynolds number was well below the turbulent threshold, ${ }^{17}$ so that our experiment was strictly in the laminar-flow regime.

The dynamic light-scattering experiment was carried out in both forward-scattering and backscattering geometries. In the forward-scattering geometry, light from a polarized He-Ne laser $(\lambda=633 \mathrm{~nm}$ ) was focused to a spot of $\simeq 30 \mu \mathrm{m}$ on one side of the cell and collected on axis with the incident beam on the other side by an optical imaging system. We measured the intensity autocorrelation function with and without a linear polarizer in the front of the photomultiplier tube used to detect the light. At $2 \%$ PSS concentration, no polarization dependence was found in the measured autocorrelation functions. This implies that the photons emerging from the cell were strongly multiply scattered. We estimate that a typical photon has scattered $\sim\left(L / l^{*}\right)^{2} \simeq 10^{2}$ times. We emphasize that no unscattered light was detected. In the backscattering geometry, the sample was illuminated by a uniform beam, $1 \mathrm{~cm}$ in diameter, from an argonion laser ( $\lambda=488 \mathrm{~nm}$ ); light was collected from a $50-\mu \mathrm{m}$ diameter spot near the center of the illuminated area on the same side of the cell by using collection optics similar to that used in forward scattering. We placed a crossed polarizer in the front of the photomultiplier tube to minimize reflection from the sample cell and single scattering. In both geometries, the strong multiple scattering ensured that the linear dimensions of the illuminated volume was in excess of $1 \mathrm{~mm}$, which is much greater than the rms displacement, $\Delta r_{\mathrm{rms}}$, of the scattering particles in one correlation time. Thus, during a measurement, the scattering particles always remained within the illuminated volume.

In the quiescent state (no flow), we measured the autocorrelation function for both forward and backward scattering. The results are shown in Fig. 3, where circles indicate backscattering and squares indicate forward scattering. To fit the data, we calculated the value for $\tau_{B}=3.18 \mathrm{msec}$ from the
Stokes-Einstein relation and checked it by DLS in the single-scattering regime. Thus the only fitting parameters were $\gamma$ and $l^{*}$. As is shown in Fig. 3, the experimental data for forward scattering and backscattering fit the theory well. From the fits, we obtained values of $\gamma=2.2$ and $l_{F}^{*}=105 \mu \mathrm{m}$ for forward scattering and $\gamma=2.2$ and $l_{B}^{*}=83 \mu \mathrm{m}$ for backscattering. The value of $\gamma$ found in this experiment was $~ 5 \%$ larger than reported in Ref. 8 owing to the different sizes of PSS's used. The difference in $l^{*}$ measured by forward scattering and backscattering in this experiment is due to the fact that we used different wavelengths of light for these two geometries. ${ }^{18,19}$ Calculations using Mie theory yield values of $l^{*}=111 \mu \mathrm{m}$ for $\lambda=633 \mathrm{~nm}$ and $l^{*}=98 \mu \mathrm{m}$ for $\lambda=488 \mathrm{~nm}$, which are within $15 \%$ of the measured values of $l^{*}$. It is interesting to note that in the multiple-scattering regime the autocorrelation function for forward scattering decays away much faster than for backscattering, even though the decay time $\tau_{B}$ for the Brownian motion of the particles is the same for both experiments. This difference is a clear demonstration of the effects of geometry: in forward scattering, only long paths can contribute to the autocorrelation function, and these decay rapidly; by contrast, in backscattering, short paths can also contribute to the autocorrelation function, and these decay much more slowly.

The same experiments were repeated with the suspension subjected to a steady shear flow. Typical results for forward scattering and backscattering, with $\bar{\Gamma}=41 \mathrm{sec}^{-1}$, are shown in Fig. 4. The solid curves through the data are fits to Eqs. (8) and (9) with $\tau_{B}, \gamma$, and $l^{*}$ set equal to the values previously obtained from measurements in the quiescent state ${ }^{20}$; thus $\tau_{S}$ is the only fitting parameter. For both geometries, the data are well described by the theory. In fact, over the entire range of accessible shear rates the measured autocorrelation functions were well fitted by Eqs. (8) and (9) with $\tau_{S}$ the only fitting parameter.

To test the validity of the relation $\tau_{S}{ }^{-1}=\bar{\Gamma} k_{0} l^{*} / \sqrt{30}$, we simultaneously measured $\tau_{S}$ using DWS and $\bar{\Gamma}$ by a direct measurement of the flow rate $J$. In Fig. 5 we plot $1 / \tau_{S} k_{0} l^{*}$

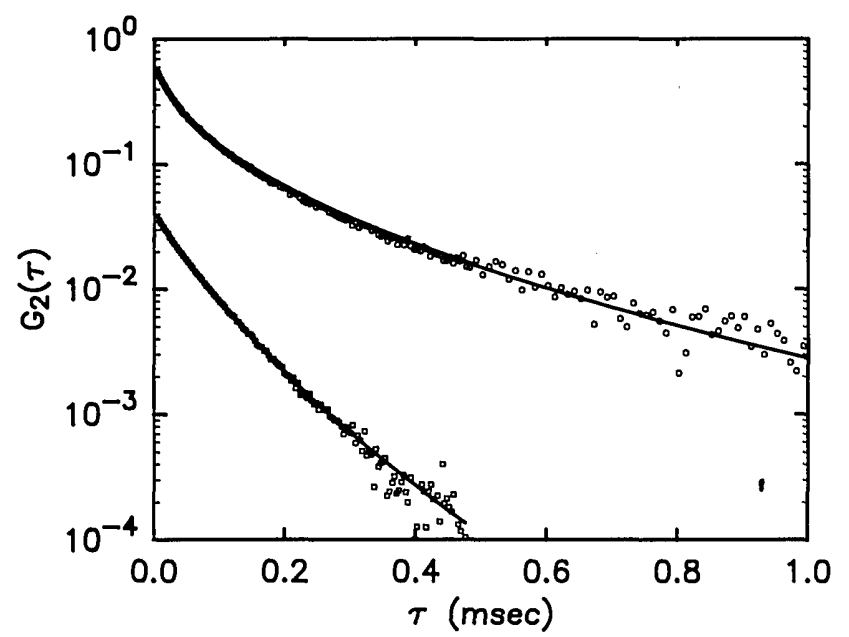

Fig. 3. $\log \left[G_{2}(\tau)\right]$ versus $\tau$ without flow. The circles (upper curve) and squares (lower curve) are experimental data for backscattering and forward scattering, respectively. The curves were fitted to the data using Eqs. (8) and (9) with Eq. (10) and letting $\tau_{S} \rightarrow \infty$. With $\gamma$ $=2.2$, we found $l_{B}^{*}=83 \mu \mathrm{m}$ and $l_{F}^{*}=105 \mu \mathrm{m}$ for backscattering and forward scattering, respectively. 


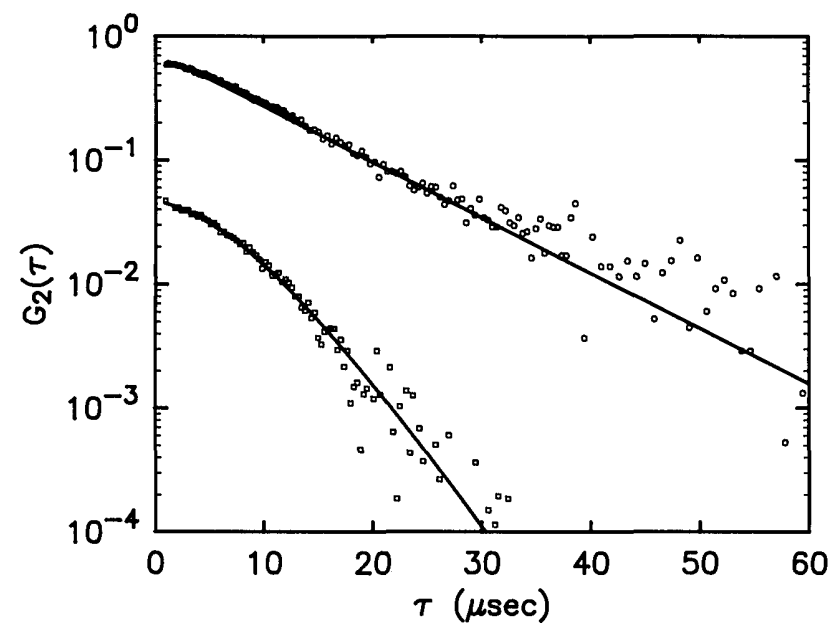

Fig. 4. $\log \left[G_{2}(\tau)\right]$ versus $\tau$ at a pumping rate of $0.165 \mathrm{~cm}^{3} / \mathrm{sec}$. The circles (upper curve) and squares (lower curve) are experimental data for backscattering and forward scattering, respectively. The curves were fitted to the data using Eqs. (9) and (10) with Eq. (10). The values of $\gamma$ and $l^{*}$ were taken from quiescent measurements, so that the only fitting parameter is $\tau_{S}$.

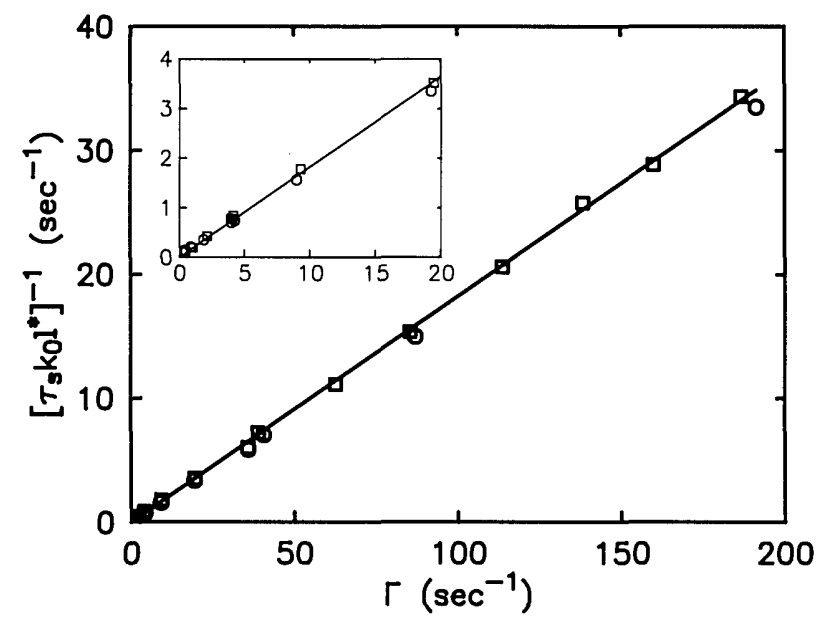

Fig. 5. $1 / \tau_{S} k_{0} l^{*}$ versus average shear rate $\bar{\Gamma}$. The meanings of the symbols are the same as in Figs. 2 and 3. For the entire range of experimentally accessible pumping rates, $0.5 \mathrm{sec}^{-1}<\bar{\Gamma}<200 \mathrm{sec}^{-1}$, our measurements agree with the theory, which is plotted as a straight line. The inset is an expanded view for low $\vec{\Gamma}$.

for both forward and backward scattering versus the shear rate $\bar{\Gamma}$. Both geometries give the same results and agree very well with the theoretically calculated slope of $1 / \sqrt{30}$, to within $10 \%$. This excellent consistency confirms that the functional forms of the autocorrelation functions are correct in spite of the drastically different time scales for the decay of the autocorrelation function in forward scattering and backscattering.

These measurements also suggest that both light-scattering geometries probe essentially the entire range of velocity gradients in the sample cell. For the transmission geometry, this will always be the case since all the light reaching the detector will have sampled the entire cross section of the cell. For the backscattering geometry, $P(s)$ decays slowly for large $s\left(\sim s^{-3 / 2}\right)$. Thus we expect that a significant fraction of the light emerging from cell will have penetrated many $l^{*}$ into the sample. Since the distance to the center of the cell in our samples is approximately $5 l^{*}$, the entire range of velocity gradients in our cells is probed in backscattering as well as in forward scattering. However, in cells that are much thicker we expect that velocity gradients deep inside the sample will not be probed since there are relatively few photons that penetrate many $l^{*}$.

For forward scattering, the autocorrelation function takes on a particularly simple form when shear dominates the decay. In the limit that $\tau_{S} \ll \tau_{B}\left(l^{*} / L\right)$,

$$
G_{1}(\tau) \simeq \frac{1}{\zeta(3)} \int_{\sqrt{6} \frac{L}{l^{*}}}^{\infty} \frac{\tau}{\tau_{S}} \frac{z^{2}}{\sinh (z)} \mathrm{d} z
$$

so that $G_{1}(\tau)$ is a function only of $\left(L / l^{*}\right)\left(\tau / \tau_{S}\right)$. Since the combination $\tau_{S} l^{*}=\sqrt{30} / \bar{\Gamma} k_{0}$ is independent of $l^{*}, G_{1}(\tau)$ should be independent of $l^{*}$ and depend only on the sample thickness $L$ and shear rate $\bar{\Gamma}$. To test this feature, we measured $G_{2}(\tau)$ at various PSS concentrations while keeping the pumping rate $J=0.15 \mathrm{~cm}^{3} / \mathrm{sec}$ fixed. In Fig. 6 we show data for four different concentrations of PSS's: $2 \%, \sim 1 \%, \sim 0.5 \%$, and $\sim 0.25 \%$, corresponding to $l^{*}=105,215,420$, and $780 \mu \mathrm{m}$, respectively. During the measurement special care was taken to minimize the contribution from single scattering, particularly at the lower PSS concentrations, by placing a crossed polarizer in the front of the photomultiplier tube. From Fig. 6 we see that all the data collapse onto a single curve, suggesting that $G_{2}(\tau)$ is indeed independent of $l^{*}$. We also measured $G_{2}(\tau)$ for a microemulsion, which was opaque and milky. ${ }^{21,22}$ Once again, we used a pumping rate of $J=0.15 \mathrm{~cm}^{3} / \mathrm{sec}$, and we again found that $G_{2}(\tau)$ was the same as in the PSS case even though the morphology of the microemulsion, and hence its scattering properties, is quite different from that of the PSS suspension. Thus we conclude that the use of DWS to measure shear rate does not depend on the details of the scattering properties of the fluid but only requires that the transport of the light be diffusive.

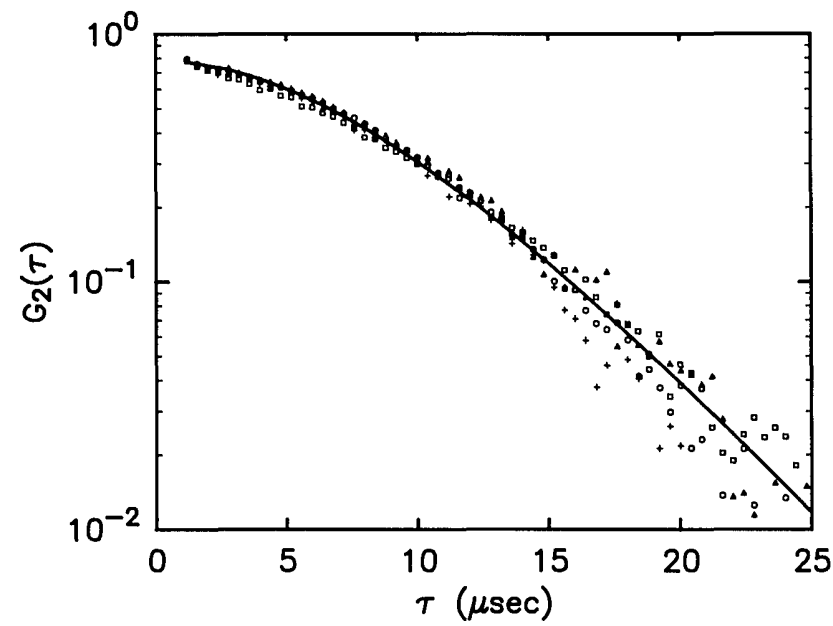

Fig. 6. $\log \left[G_{2}(\tau)\right]$ versus $\tau$ for measurement at the same pumping rate as in Fig. 5 but with different PSS concentrations. The measurements were made in the forward-scattering geometry with a pumping rate of $0.15 \mathrm{~cm}^{3} / \mathrm{sec}$. The PSS's at volume fractions of $2 \%$ (squares), $1 \%$ (triangles), $0.5 \%$ (circles), and $0.25 \%$ (crosses) were used. Fits to Eq. (9) gave $l^{*}=105,215,420$, and $780 \mu \mathrm{m}$, respectively for the same concentrations given above. As is indicated, $G_{2}(\tau)$ was independent of $l^{*}$, in agreement with the theory. 


\section{CONCLUSIONS}

We have developed a simple theory for obtaining the functional form of the autocorrelation functions in the multiplescattering regime for Brownian particles exposed to a laminar shear flow. The key to the theory is the description of the transport of light as a diffusive process. In our experiments the dynamical time scales associated with the motion of the scatterers were varied by more than 2 orders of magnitude, from the longest free diffusion time, $\tau_{B} \simeq 3 \mathrm{msec}$, to the shortest shear time, $\tau_{S} \simeq 20 \mu$ sec. The experimental results for both forward scattering and backscattering are well fitted by the theory over the entire dynamical range. For forward scattering we found that autocorrelation function is independent of particle shape, size, concentration, and polydispersity. Thus, to measure the mean shear rate one only needs to know the sample thickness and wavelength used. This unique feature of forward scattering should be useful in many practical applications.

These results illustrate the potential power of DWS for studying flow in systems in which multiple scattering is unavoidable. Thus DWS extends the applicability of DLS to many previously inaccessible systems, including dense colloidal suspensions, microemulsions, and porous media.

\section{ACKNOWLEDGMENTS}

We thank Penger Tong, Daniel Ou-Yang, and Fred MacKintosh for stimulating discussions and M. W. Kim for a generous loan of his equipment.

P. M. Chaikin is also with the Department of Physics, Princeton University, Princeton, New Jersey 08540.

\section{REFERENCES AND NOTES}

1. G. G. Fuller, J. M. Rallison, R. L. Schmidt, and L. G. Leal, "The measurement of velocity gradients in laminar flow by homodyne light-scattering spectroscopy," J. Fluid Mech. 100, 555 (1980).

2. P. Tong, W. I. Goldburg, C. K. Chan, and A. Sirivat, "Turbulent transition by photon-correlation spectroscopy," Phys. Rev. A 37, 2125 (1988).

3. P. M. Chaikin, J. M. di Meglio, W. D. Dozier, H. M. Lindsay, and D. A. Weitz, in Physics of Complex and Supermolecular Fluids (Wiley, New York, 1987), p. 65.

4. P. Pieranski, "Colloidal crystals," Contemp. Phys. 24, 25 (1983).

5. R. Bonner and R. Nossal, "Model for laser Doppler measurements of blood flow in tissue," Appl. Opt. 20, 2097 (1981).
6. A. Ishimaru, Wave Propagation in Random Media (Academic, New York, 1978).

7. G. Maret and P. E. Wolf, "Multiple light scattering from disordered media: the effect of Brownian motion of scatterers," $Z$. Phys. B 65, 409 (1987).

8. D. J. Pine, D. A. Weitz, P. M. Chaikin, and E. Herbolzheimer, "Diffusing-wave spectroscopy," Phys. Rev. Lett. 60, 1134 (1988).

9. F. C. MacKintosh and S. John, Phys. Rev. B 40, 2383 (1989).

10. The correction to Brownian motion that is due to the convective flow is called Taylor dispersion. Taylor dispersion modifies particle diffusion in the direction of velocity gradient. In the entire range of shear rate in this experiment the correction $(\Gamma \tau)^{2} / 3$ is much smaller than 1 . This justifies our approximation that the particle diffusion and convective shear are decoupled.

11. For some scattering geometries there will be an additional term in the sum corresponding to the difference between the input and output wave vectors. This term is proportional to the velocity (rather than to the velocity gradient) and does not contribute to the homodyne correlation function. We also note that for the common case that the flow direction is perpendicular to the input and output wave vectors, this term is identically zero.

12. G. K. Batchelor, An Introduction to Fluid Dynamics (Cambridge U. Press, Cambridge, 1977), p. 83.

13. More generally, to include the effects of particle interactions, one must replace $F(q)$ by the full scattering function $S(q) F(q)$, where $S(\mathbf{q})$ is the structure factor. We note that in these experiments, however, the volume fraction of PSS's is low ( $\phi=0.02)$ and the Coulomb interaction between spheres is highly screened. Under these conditions, $S(\mathbf{q}) \simeq 1$.

14. B. Chu, Laser Light Scattering (Academic, New York, 1974), pp. 101-104.

15. L. D. Landau and E. M. Lifshitz, Fluid Mechanics (Pergamon, Oxford, 1984), p. 55

16. Carboxylated polystyrene spheres were purchased from Duke Scientific, Palo Alto, California.

17. D. J. Tritton, Physical Fluid Dynamics, 2nd ed. (Clarendon, Oxford, 1988), p. 20.

18. P. E. Wolf, G. Maret, E. Akkermans, and R. Maynard, "Optical coherent backscattering by random media: an experimental study," J. Phys. (Paris) 49, 63 (1988).

19. E. Akkermans, P. E. Wolf, R. Maynard, and G. Maret, "Theoretical study of the coherent backscattering of light by disordered media," J. Phys. (Paris) 49, 77 (1988).

20 . We note that the average intensity of transmitted light, $\langle I\rangle$, through the sample did not vary with $\bar{\Gamma}$. Since $\langle I\rangle \sim l^{*} / L$, we conclude that $l^{*}$, and hence $P(s)$, does not vary with $\bar{\Gamma}$ for our samples (see Refs. 6 and 8). This also suggests that $S(q)$ is essentially independent of $\Gamma$ for the weakly interacting samples used in this study.

21. J. S. Huang and M. W. Kim, "Critical behavior of a microemulsion," Phys. Rev. Lett. 47, 1462 (1981).

22. S. H. Chen, T. L. Lin, and J. S. Huang, in Physics of Complex and Supermolecular Fluids (Wiley, New York, 1987), p. 285. 